\title{
Statistical properties of partially coherent CW fiber lasers
}

\author{
Dmitriy V. Churkin ${ }^{* a, b}$, Sergey V. Smirnov ${ }^{b}$, Evgenii V. Podivilov ${ }^{a}$ \\ a Institute of Automation and Electrometry, SB RAS, Novosibirsk 630090, Russia; \\ ${ }^{\mathrm{b}}$ Novosibirsk State University, Novosibirsk, 630090, Russia
}

\begin{abstract}
A detailed quantitative numerical analysis of partially coherent quasi-CW fiber laser is performed on the example of high-Q cavity Raman fiber laser. The key role of precise spectral performances of fiber Bragg gratings forming the laser cavity is clarified. It is shown that cross phase modulation between the pump and Stokes waves does not affect the generation. Amplitudes of different longitudinal modes strongly fluctuate obeying the Gaussian distribution. As intensity statistics is noticeably non-exponential, longitudinal modes should be correlated.
\end{abstract}

Keywords: fiber lasers; partially coherent radiation; propagation in fibers; nonlinear optics; photon statistics; modes correlations.

\section{INTRODUCTION}

The output radiation of conventional partially coherent (PC) continuous-wave (CW) fiber lasers consists of numerous longitudinal modes. Typically, ytterbium-doped fiber laser (YDFL) supports simultaneous generation of $\sim 10^{3}$ longitudinal modes, while in Raman fiber lasers (RFLs) modes number can be up to $10^{8}$ [1]. Simple laser models based on balance equations ${ }^{2}$ can not predict generation properties of PC fiber lasers since four wave-mixing (FWM) interactions of numerous longitudinal modes induce spectral broadening which affects sufficiently the mirror reflection and, thus, output power. Complex numerical modeling based on non-linear Schrödinger equation (NLSE) can provide both power and generation spectrum as well as time dynamics of PC CW fiber sources of different types ${ }^{3-7}$.

Fundamentally, statistical properties of laser radiation are of great interest. However, statistical properties have been only studied for stimulated Brillouin scattering based $\mathrm{CW}$ fiber lasers ${ }^{8}$. At the same time, contradictory phenomenological models of PC quasi-CW laser radiation statistics are used. For example, PC radiation is represented as pure $\mathrm{CW}$ single longitudinal mode beam without any intensity and phase fluctuations ${ }^{9}$. Authors of papers ${ }^{10,11}$ assume constant timedomain field having phases correlated to obtain Lorentzain generation spectrum, while different spectral modes of random $\delta$-correlated phases and fixed amplitudes are treated in papers ${ }^{12}{ }^{13}$. It is known that results of modeling strongly depend on the statistics of the input initial PC field ${ }^{6,13}$ in problems of supercontinuum generation and PC field propagation.

\section{NUMERICAL METHOD}

In the present paper we provide a detailed description including statistical properties of the PC quasi-CW fiber laser using the high-Q normal dispersion cavity RFL as a test bed for our methods. To model a laser generation we use 4 coupled NLSEs ${ }^{14}$ :

$$
\begin{gathered}
\frac{\partial A_{p}^{ \pm}}{\partial z}+\frac{i}{2} \beta_{2 p} \frac{\partial^{2} A_{p}^{ \pm}}{\partial t^{2}}+\frac{\alpha_{p}}{2} A_{p}^{ \pm}=i \gamma_{p}\left(\left|A_{p}^{ \pm}\right|^{2}+2\left|A_{s}^{ \pm}\right|^{2}\right) A_{p}^{ \pm}-\frac{g_{p}}{2}\left(\left|A_{s}^{ \pm}\right|^{2}+\left\langle\left|A_{s}^{\mp}\right|^{2}\right\rangle\right) A_{p}^{ \pm} \\
\frac{\partial A_{s}^{ \pm}}{\partial z}+\left(\frac{1}{v_{s}}-\frac{1}{v_{p}}\right) \frac{\partial A_{s}^{ \pm}}{\partial t}+\frac{i}{2} \beta_{2 s} \frac{\partial^{2} A_{s}^{ \pm}}{\partial t^{2}}+\frac{\alpha_{s}}{2} A_{s}^{ \pm}=i \gamma_{s}\left(\left|A_{s}^{ \pm}\right|^{2}+2\left|A_{p}^{ \pm}\right|^{2}\right) A_{s}^{ \pm}+\frac{g_{s}}{2}\left(\left|A_{p}^{ \pm}\right|^{2}+\left\langle\left|A_{p}^{\mp}\right|^{2}\right\rangle\right) A_{s}^{ \pm}
\end{gathered}
$$

*churkin@iae.nsk.su; phone 7383332 82 54; fax 73833328254.

Nonlinear Frequency Generation and Conversion: Materials, Devices, and Applications X, edited by Konstantin L. Vodopyanov, Proc. of SPIE Vol. 7917, 79171X · @ 2011 SPIE

CCC code: $0277-786 \mathrm{X} / 11 / \$ 18 \cdot$ doi: $10.1117 / 12.877672$ 
where $\mathrm{A}$ is complex field envelope, $\mathrm{z}$ is a coordinate, $\mathrm{t}$ stands for time in a frame of references moving with pump wave, $\mathrm{v}_{\mathrm{s}}$ and $\mathrm{v}_{\mathrm{p}}$ are pump and Stokes waves group-velocities, $\beta_{2}$ and $\alpha$ describe dispersion and linear attenuation, $\gamma$ and $g$ are Kerr and Raman coefficients that are inversely proportional to wavelength. Indices \pm denote counter-propagating waves, "s" and "p" are used for Stokes and pump waves. We integrated Eq. (1) along z using iterative approach, i.e. when integrating equations for $\mathrm{A}_{\mathrm{s}, \mathrm{p}}^{+}$we used $\mathrm{A}_{\mathrm{s}, \mathrm{p}}$ obtained on previous iteration, and vice versa. The similar approach has been used previously for modeling fiber lasers, see, for example ${ }^{8,15}$. Pumping was added to $A_{p}$ at the beginning of each roundtrip (i.e. before modeling of light propagation in "+"-direction). A white noise (1 photon in each longitudinal mode ${ }^{16}$ ) was used as initial condition for Stokes wave what modeled spontaneous Raman emission. The generation becomes stable after $10^{2}-10^{4}$ round-trips depending on the pump power. In our simulations we used time windows as wide as 3-50 ns and grid with $2^{12}-2^{16}$ points with inverse mesh width of $\sim 6 \mathrm{~nm}$. We have checked that nor decreasing the time steps, nor increasing the time window, nor decreasing integration step $\Delta z$, nor changing initial white noise intensity affect the results. Intensities of counter-propagating waves enter to equations only by means of their average values since these waves move fast with regard to each other. Moreover so do the co-propagating pump and Stokes waves. Indeed, for typical spectral width of $1 \mathrm{~nm}$ Stokes waves oscillate with a period of $\sim 3.5 \mathrm{ps}$. Due to different group velocities waves walk-off is occurred on $\mathrm{L}_{\mathrm{w}} \sim 1 \mathrm{~m}$ of fiber. In contrast, for $\mathrm{P} \sim 2.5 \mathrm{~W}$ nonlinear length $\mathrm{L}_{\mathrm{NL}} \sim 100 \mathrm{~m}$ what is much longer that walk-off length. It makes one possible to average Eq. (1) over multiple $\mathrm{L}_{\mathrm{w}}$ scales. As a result, XPM term leads to time-independent phase shift only and therefore can be omitted. This fact makes one possible to integrate Eq (1) using a much longer integration step $\Delta z$, what speed up simulations significantly. Below we will discuss the role of XPM in more details.

\section{POWER AND SPECTRAL PROPERTIES}

We have found that the generated power as well as spectrum are sensitive to the FBGs performances. In particular, varying the width of the modelled super-gaussian FBGs by only $10 \%$, the generation power is changed, Fig. 1a. The power and the spectrum strongly depend also on a relative difference of FBGs central frequencies, Fig. 1a.
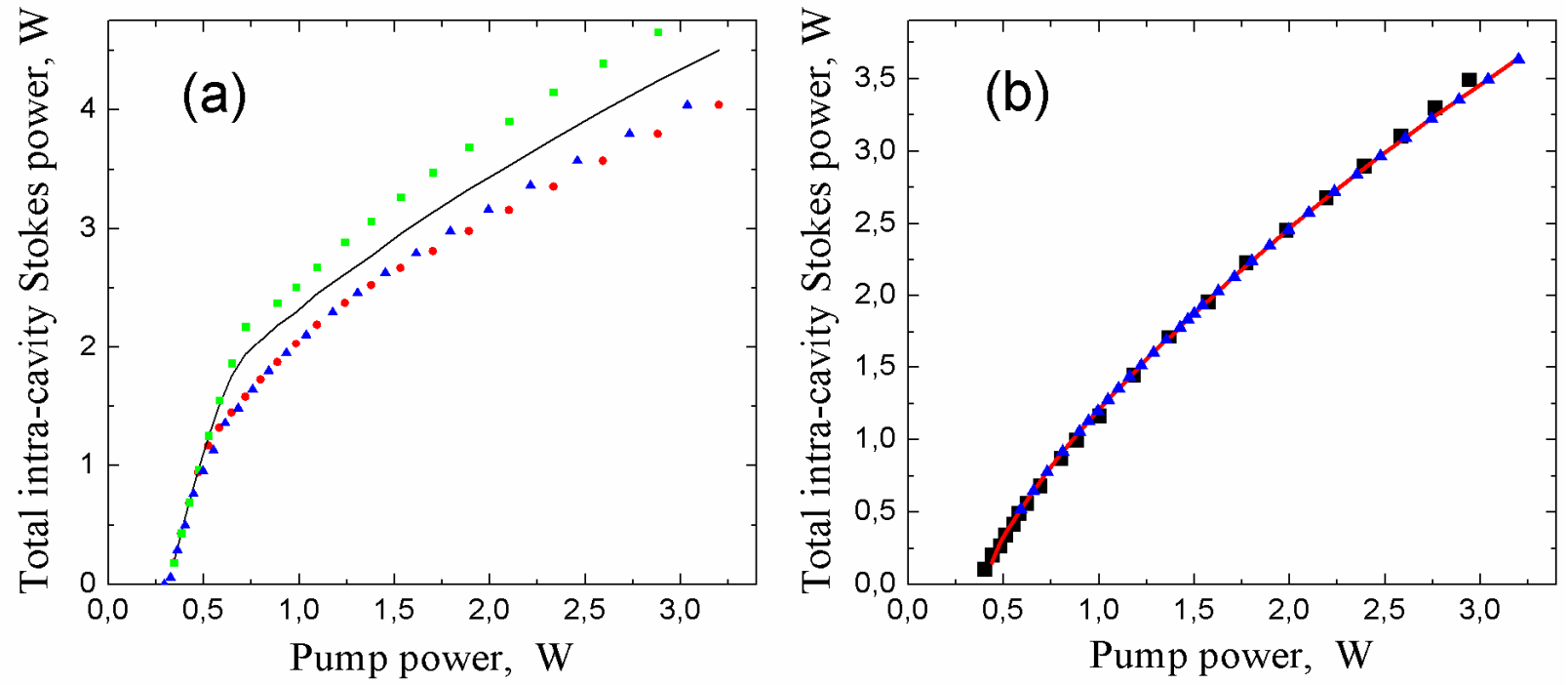

Figure 1. (Color online) (a) Total generated intra-cavity power depending on FBGs width (1.0 nm - line, $1.1 \mathrm{~nm}$ boxes, $0.9 \mathrm{~nm}$ - circles) and relative difference of FBGs central frequencies $(0 \mathrm{~nm}$ - line, $0.2 \mathrm{~nm}$ - triangles). (b) Total generated intra-cavity power: experimental $\operatorname{dots}^{17}$, numerically calculated without XPM (line) and with XPM (triangles). 


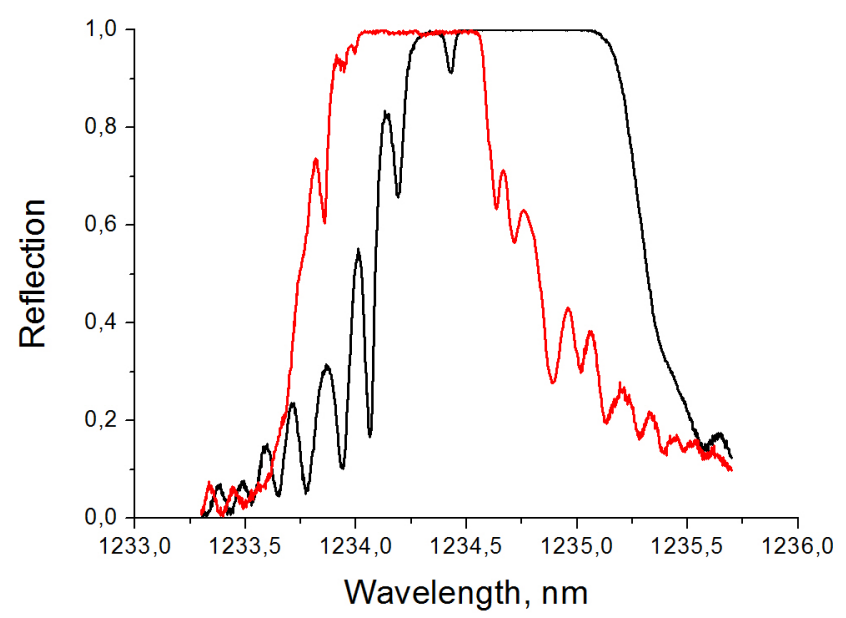

Figure 2. Spectral profiles of FBGs used in the experiment ${ }^{17}$ and the presented modeling.

As a result, for an accurate modelling even of such integral characteristic as generated power one should use the experimentally measured FBGs profiles which are usually far away from any simple analytical function and have wellpronounced ripples. Further we have used real FBG profiles from ${ }^{17}$ which are relatively de-centered by $\sim 0.5 \mathrm{~nm}$ and have spectral width of $0.7 \mathrm{~nm}$ and $1 \mathrm{~nm}$, see Fig. 2. Values of all coefficients were also taken from ${ }^{17}: \alpha_{\mathrm{p}}=0.5(\mathrm{~km})^{-1}, \alpha_{\mathrm{s}}$ $=0.83(\mathrm{~km})^{-1},\left(\alpha_{\mathrm{p}}, \alpha_{\mathrm{s}}\right.$ values include lumped losses on splices, couplers etc. $), \beta_{2 \mathrm{p}}=17.9 \mathrm{ps}^{2} / \mathrm{km}, \beta_{2 \mathrm{~s}}=7.17 \mathrm{ps}^{2} / \mathrm{km}, \gamma_{\mathrm{s}}=3$ $(\mathrm{km} * \mathrm{~W})^{-1}, \mathrm{~g}_{\mathrm{s}}=1.3\left(\mathrm{~km}^{*} \mathrm{~W}\right)^{-1}$, the cavity length $\mathrm{L}=370 \mathrm{~m}$.
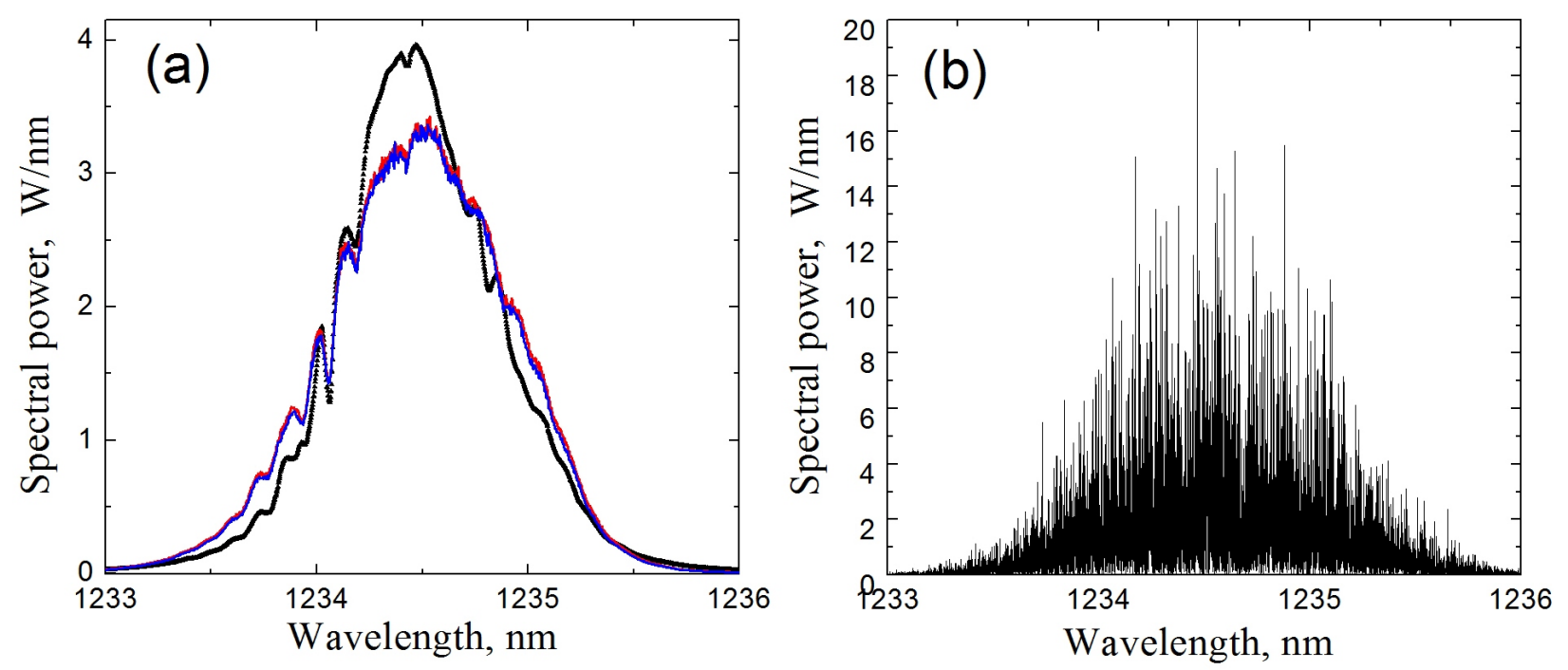

Figure 3. (Color online) (a) Intra-cavity spectrum at 3.5W: experimental dots, calculated without XPM using multimode (blue line) or SF (red line) initial pump conditions. Taking into account XPM provides spectra indistinguishable from shown. (b) The spectrum calculated without XPM at 3.5 W (the same as at panel (a), blue line) but without averaging at many realizations.

The generated total intra-cavity Stokes wave power agrees well with the experimental data, Fig. 1b. An averaged over 400 full passes and $0.01 \mathrm{~nm}$ optical spectrum is also in good agreement with experimental one, Fig. 3a. However, the spectral broadening in the modelling is more pronounced. The spectrum is highly sensitive to the dispersion and nonlinearity values, but in ${ }^{17}$ fiber coefficient are defined with accuracy no better than $15 \%$. Lower $\beta_{2 s}$ allows interaction through FWM processes of larger number of modes, as well as higher $\gamma_{s}$ makes FWM processes more intense thus increasing the spectral broadening. Changing $\beta_{2 s}, \gamma_{s} g_{s}, \alpha$ within its accuracy, one can preserve good coincidence for the 
generated power, and provide at the same time good coincidence of the calculated and experimentally measured spectra. Thus, for precise calculation of laser performances, one needs to know not only experimental FBG profiles, but also exact values of fiber parameters

The pump radiation is provided by YDFL exhibiting strong intensity fluctuations. The XPM-induced noise transfer from pump to Stokes wave is experimentally observed in radio frequency beating spectrum ${ }^{18}$. However we revealed that XPM has no influence on laser performances except near the threshold. Indeed, we can turn out the term responsible for XPM between pump and Stokes wave in the Eqs. (1). Equal generated power values, the same spectral width values, and fully identical spectral shapes in simulations with and without XPM, see Fig.1b, Fig.3a, prove that XPM processes are not responsible for the spectrum formation and broadening. Indeed, the typical nonlinear length LNL $\sim 100 \mathrm{~m}$ is much longer that walk-off length $\mathrm{Lw} \sim 1 \mathrm{~m}$ related with group velocities vs, vp difference. If $\mathrm{L}_{\mathrm{w}}<<\mathrm{L}_{\mathrm{NL}}$ it is possible to average Eqs. (1) over length La being $\mathrm{L}_{\mathrm{w}}<<\mathrm{L}_{\mathrm{a}}<<\mathrm{L}_{\mathrm{NL}}$. So XPM term leads to time independent phase incursion only, and therefore does not contribute to spectral broadening and can be omitted. We have also tested that spectral width of multimode pump has no impact on generation. Moreover, even single frequency (SF) pump without any amplitude and phase fluctuations provides the identical generated power and spectra with those provided by multimode wave, Fig.3a.
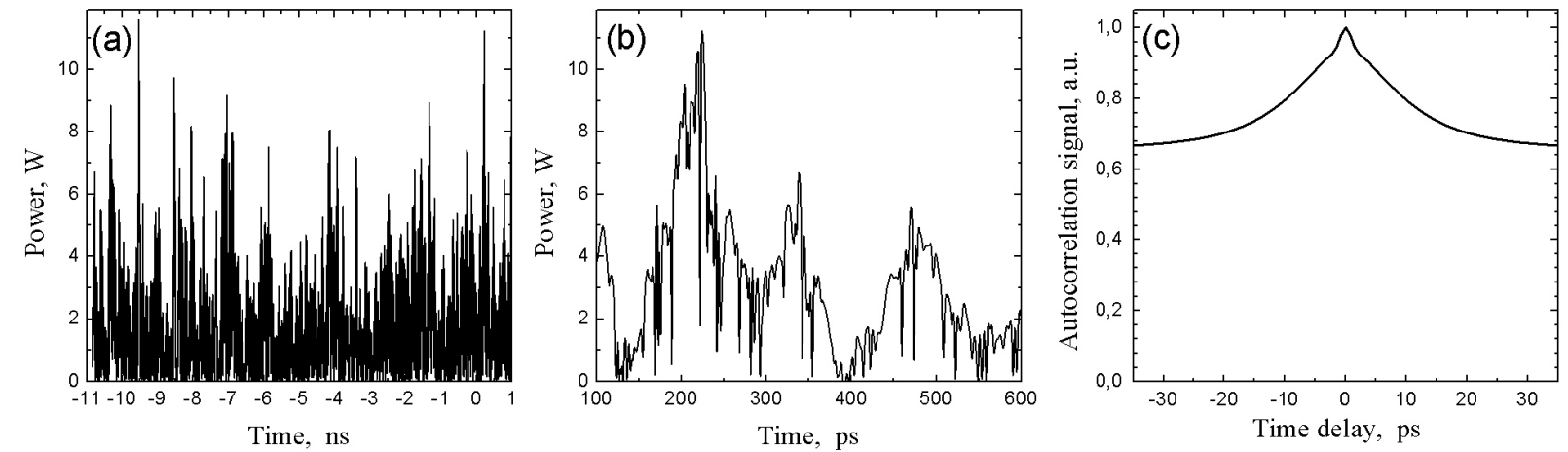

Figure 4. (a-b) Typical time dynamics at $3.5 \mathrm{~W}$ at different timescales. (c) Auto-correlation function at $3.5 \mathrm{~W}$.

\section{TIME DYNAMICS AND STATISTICAL PROPERTIES}

Fast time dynamics of intensity is also calculated. Typically, stochastic pulses of different width and amplitudes varying from zero to the value several times higher then the mean are generated, Fig.4a. The zoomed in time dynamics clearly reveal that intensity can dropped down to zero, Fig.4b. Averaged over many realizations autocorrelation function (ACF), Fig. 4c, corresponds to the time dynamics by the typical timescale and qualitatively coincides with the measured $\mathrm{ACF}^{12}$.

Finally, we have carefully analyzed intensity, spectral density and phase statistics. The generation spectrum before averaging reveals strong intensity fluctuations, Fig.3b. The maximum spectral power density can be several times higher than averaged over many realizations (or over finite measurement time in experiment) spectral power density. Probability density function (PDF) of the spectral power density defined from the non-averaged generated spectra is exponential (i.e. Gaussian for amplitudes of longitudinal modes) and does not depend on power, Fig. 5a. Thus the amplitudes of longitudinal modes can not be treated as fixed as it assumed in the phenomenological models of PC radiation of fiber lasers ${ }^{9-13}$. We have checked that the phases of different longitudinal modes are distributed uniformly within the numerics accuracy, Fig. 5c.

The consideration of the numerous modes as statistically independent with random $\delta$-correlated phases and Gaussian distributed fluctuating amplitudes must leads through the central limit theorem to the exponential PDF for intensity I(t), $\mathrm{P}[\mathrm{I}(\mathrm{t})] \sim \exp (-\mathrm{I}(\mathrm{t}) /<\mathrm{I}>)$. However, the intensity PDF obtained from numerous time traces is noticeably non-exponential and depends on power, Fig. 5b. Therefore we conclude that generation modes have to be noticeably correlated. We carefully checked that pump wave initial conditions does not affect statistical properties. 

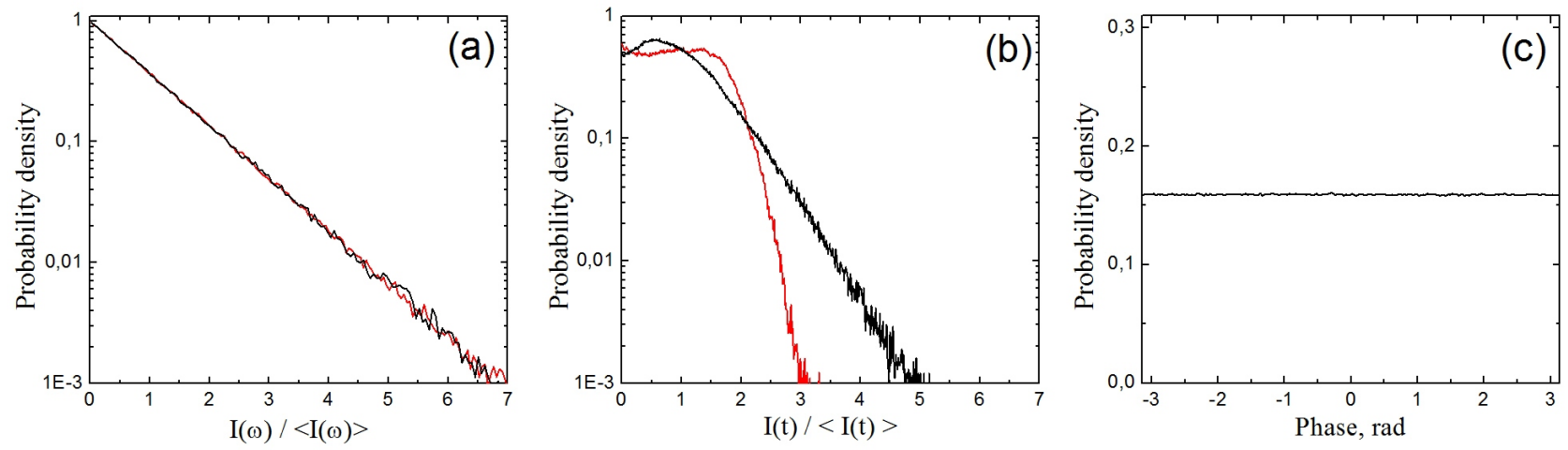

Figure 5. (Color online) (a) Spectral power density PDF. (b) Intensity PDF at $0.11 \mathrm{~W}$ (red) and $3.5 \mathrm{~W}$ (black) of generated power. (c) Phase distribution.

\section{CONCLUSIONS}

To conclude, the direct NLSE based numerical modelling of the partially coherent quasi-CW fiber laser is performed on the example of the high-Q normal dispersion cavity RFL. The good quantitative agreement with experimental data for power and spectral performances is obtained. It is shown that precise spectral profiles of FBGs forming the laser cavity influence sufficiently real laser performances. We prove that the generation is not sensitive to the XPM between the pump and generated waves. The amplitudes of different modes strongly fluctuate having Gaussian PDF. Amplitudes fluctuations should be taken into account while modelling the PC field of quasi-CW fiber laser output for using as initial conditions in other problems such as supercontinuum generation and radiation propagation in fibers. The intensity exhibits strong fast fluctuations with non-exponential and power dependent PDF revealing that longitudinal modes are correlated. Note that the spectral and statistical properties might be different in anomalous dispersion cavity ${ }^{19}$.

The implemented iterative approach of solving boundary problem can be used for modelling and optimization of other types of PC quasi-CW fiber lasers (Yb-, Er-doped or ultra-long RFLs) after corresponding modification of the gain type in Eqs.(1). A big challenge is to implement the approach for describing of recently developed random distributed feedback (DFB) fiber lasers ${ }^{20}$ operating via Raman-amplified distributed Rayleigh backscattering. Similar to demonstration of strong intensity fluctuations suppression in Rayleigh scattering assisted SBS fiber laser ${ }^{8}$, the random DFB fiber laser could have intensity fluctuations lower than traditional PC Raman fiber sources.

The authors acknowledge support from the Russian Ministry of Science and Education, and thank A.A. Fotiadi, S.A. Babin, M.P. Fedoruk for fruitful discussions. The computations were performed at the Novosibirsk State University Scientific computing center.

\section{REFERENCES}

[1] Babin, S., A., Karalekas, V., Podivilov, E., V., Mezentsev, V., K., Harper, P., Ania-Castañón J., D., and Turitsyn, S., K., "Turbulent broadening of optical spectra in ultralong Raman fiber lasers", Physical Review A 77,1 (2008).

[2] Auyeung, J., and Yariv, A., "Theory of CW Raman oscillation in optical fibers”, J. Opt. Soc. Am. 69 803, (1979)

[3] Vanholsbeeck, F., Coen, S., Emplit, P., Martinelli, C., Leplingard, F., and Sylvestre, T., "Numerical modeling of a four-wave-mixing-assisted Raman fiber laser”, Opt. Lett. 29, 2719 (2004).

[4] Turitsyna, E., G., Turitsyn, S., K., and Mezentsev, V., K., "Numerical investigation of the impact of reflectors on spectral performance of Raman fibre laser", Opt. Express 18, 4469 (2010).

[5] Roy, V., Babin, P., M, F., and Schinn, G., "Nonlinear wave mixing in a multilongitudinal mode erbium-doped fiber laser", Opt. Express 13, 6791 (2005). 
[6] Travers, J., C., Popov, S., V., and Taylor, J., R., "A new model for CW supercontinuum generation," in Conference on Lasers and Electro-Optics/Quantum Electronics and Laser Science Conference and Photonic Applications Systems Technologies, OSA Technical Digest (CD) (Optical Society of America, 2008), paper CFC2.

[7] Dalloz, N., Randoux, S., and Suret, P., "Influence of dispersion of fiber Bragg grating mirrors on formation of optical power spectrum in Raman fiber lasers", Opt. Lett. 35, 2505 (2010).

[8] Fotiadi, A., A., and Kiyan, R., V., "Cooperative stimulated Brillouin and Rayleigh backscattering process in optical fiber", Optics letters 23, 1805 (1998).

[9] Kobtsev, S., and Smirnov, S., "Modelling of high-power supercontinuum generation in highly nonlinear, dispersion shifted fibers at CW pump", Opt. Express 13, 6912 (2005).

[10] Mussot, A., Lantz, E., Maillotte, H., Sylvestre, T., Finot, C., and Pitois, S., "Spectral broadening of a partially coherent CW laser beam in single-mode optical fibers", Opt. Express 12, 2838 (2004).

[11] Frosz, M., Bang O., and Bjarklev, A., "Soliton collision and Raman gain regimes in continuous-wave pumped supercontinuum generation", Opt. Express 14, 9391 (2006)

[12] Vanholsbeeck, F., Martin-Lopez, S., González-Herráez, M., and Coen, S., "The role of pump incoherence in continuous-wave supercontinuum generation", Opt. Express 13, 6615 (2005)

[13] Barviau, B., Randoux, S., and Suret, P., "Spectral broadening of a multimode continuous-wave optical field propagating in the normal dispersion regime of a fiber", Opt. Lett. 31, 1696 (2006).

[14] Agrawal, G., P., Nonlinear fiber optics, 3d edition, Academic Press, 2001

[15] Latkin, A., I., Kurkov, A., S., Turitsyn, S., K.,"Spectral broadening and power leakage in CW Yb-doped fibre laser", CLEO/Europe - EQEC 2009, Conference Digest, Munich. 2009, P. CJ.P.31 THU.

[16] Smith R.G. "Optical power handling capacity of low loss optical fibers as determined by stimulated Raman and Brillouin scattering", Applied Optics, 11, 2489 (1972).

[17] Babin, S., A., Churkin, D., V., Ismagulov, A., E., Kablukov, S., I., Podivilov, E., V., "Four-wave-mixinginduced turbulent spectral broadening in a long Raman fiber laser", J. Opt. Soc. Am. A 24, 1729 (2007).

[18] Babin, S., A., Churkin, D., V., Fotiadi, A., A., Kablukov, S., I., Medvedkov, O., I., and Podivilov, E.,V., "Relative intensity noise in cascaded Raman fiber lasers", IEEE Phot. Techn. Lett. 17, 2553 (2005).

[19] Turitsyna, E., G., Falkovich, G., Mezentsev, V., K., and Turitsyn, S., K., "Optical turbulence and spectral condensate in long-fiber lasers", Phys. Rev. A 80, 031804(R) (2009).

[20] Turitsyn, S., K., Babin, S., A., El-Taher, A., E., Harper, P., Churkin, D., V., Karalekas, V., Podivilov, E., V., Kablukov, S., I., and Ania-Castanon, J., D., "Random distributed feedback fibre laser", Nature Photonics 4, 231 (2010). 